This item was submitted to Loughborough's Research Repository by the author.

Items in Figshare are protected by copyright, with all rights reserved, unless otherwise indicated.

\title{
Promoting stair climbing in public-access settings: an audit of intervention opportunities in England
}

PLEASE CITE THE PUBLISHED VERSION

http://dx.doi.org/10.1016/j.ypmed.2011.08.024

PUBLISHER

(C) Elsevier Inc.

VERSION

AM (Accepted Manuscript)

LICENCE

CC BY-NC-ND 4.0

\section{REPOSITORY RECORD}

Webb, Oliver J., and Lee Smith. 2019. "Promoting Stair Climbing in Public-access Settings: An Audit of Intervention Opportunities in England". figshare. https://hdl.handle.net/2134/9030. 
This item was submitted to Loughborough's Institutional Repository (https://dspace.lboro.ac.uk/) by the author and is made available under the following Creative Commons Licence conditions.

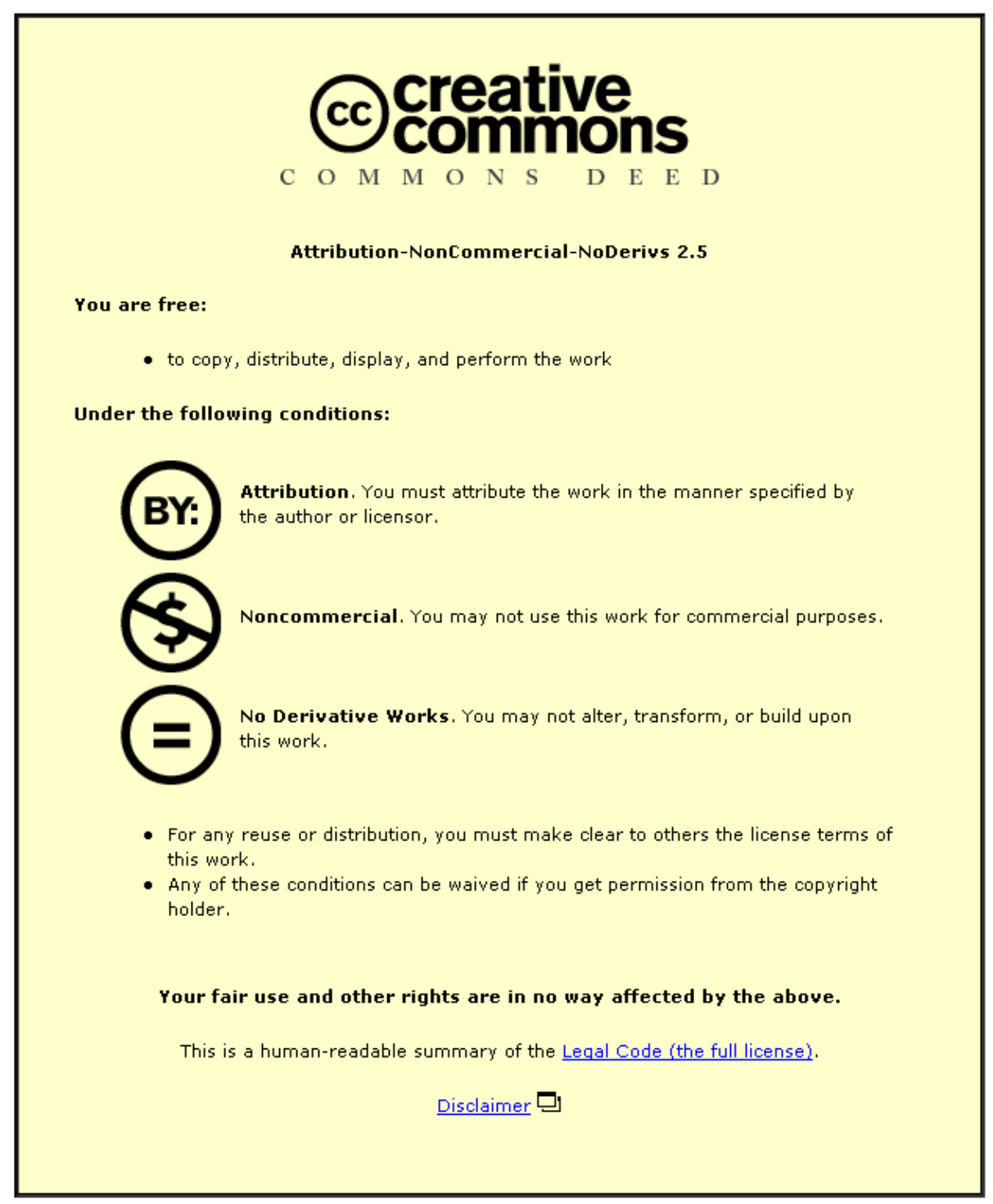

For the full text of this licence, please go to: http://creativecommons.org/licenses/by-nc-nd/2.5/ 
Promoting stair climbing in public-access settings; an audit of intervention opportunities in England

\author{
Oliver J. Webb*, Lee Smith
}

Preventive Medicine, 2011; 53: 321-324

*corresponding author 


\section{ABSTRACT}

\section{Objective}

Introducing message prompts at the 'point-of-choice' (POC) between stairs and escalators increases stair choice in 'public-access' settings (e.g. malls). For nationwide campaigns, plentiful POCs appear needed. We audited the availability of POCs in public-access settings across England.

\section{Methods}

Boundaries for 25 urban areas (population=6,829,874) were verified using Ordinance Survey maps, which showed all airports and train/tram stations. Malls and bus stations were identified from commercial listings and local authority web-pages. From September 2010March 2011 two investigators visually inspected all venues $(\mathrm{N}=410)$, counting 'true' POCs and 'quasi’ POCs (i.e. instances where stairs were visible from, but not adjacent to, escalators).

\section{Results}

$5 \%$ of venues had $\geq 1$ true POC (quasi POC $=3 \%$ ). Aggregating across areas, there was a true and quasi POC for every 243,924 and 379,437 people, respectively. There were regional variations; one area had 10 true/quasi POCs, whilst 10/24 remaining areas had none. POCs were more common in airports (4/6 venues) than malls (11/85) and train stations (4/215).

\section{Conclusion}

Although public-access POCs reach sizeable audiences, their availability in England is sporadic, precluding nationwide campaigns. Interventions should be considered locally, based on available POCs. Work/community venues (e.g. offices, hospitals), where pedestrians choose between stairs and elevators, may provide greater intervention opportunities. 


\section{Introduction}

Taking stairs, to make routine journeys between floors, can benefit individuals' health (Meyer et al., 2010). Furthermore, several barriers to participation do not apply (e.g. weather, expense, attire). 40+ studies have sought to promote stair choice, through introducing prompts at the 'point-of-choice' (POC) between stairs and the mechanical alternative (Figure 1) [Nocon et al., 2010]. Interventions take place in two distinct settings: 'public-access' venues (i.e. malls, airports, bus/train/tram stations) and work/community settings (i.e. offices, universities, libraries, hospitals). In public-access venues, fewer pedestrians use elevators than more readily available escalators (7.1\% vs. 84.0\%, Webb et al. unpublished). Accordingly, all public-access interventions to-date involved stair/escalator POCs. Work/community settings typically feature multiple storeys and few escalators. Here, elevator use is the target behaviour, often accounting for $>80 \%$ of ascents (Bungum et al., 2007). Consequently, all work/community interventions bar one involved stairs/elevator POCs (Coleman and Gonzalez, 2001).

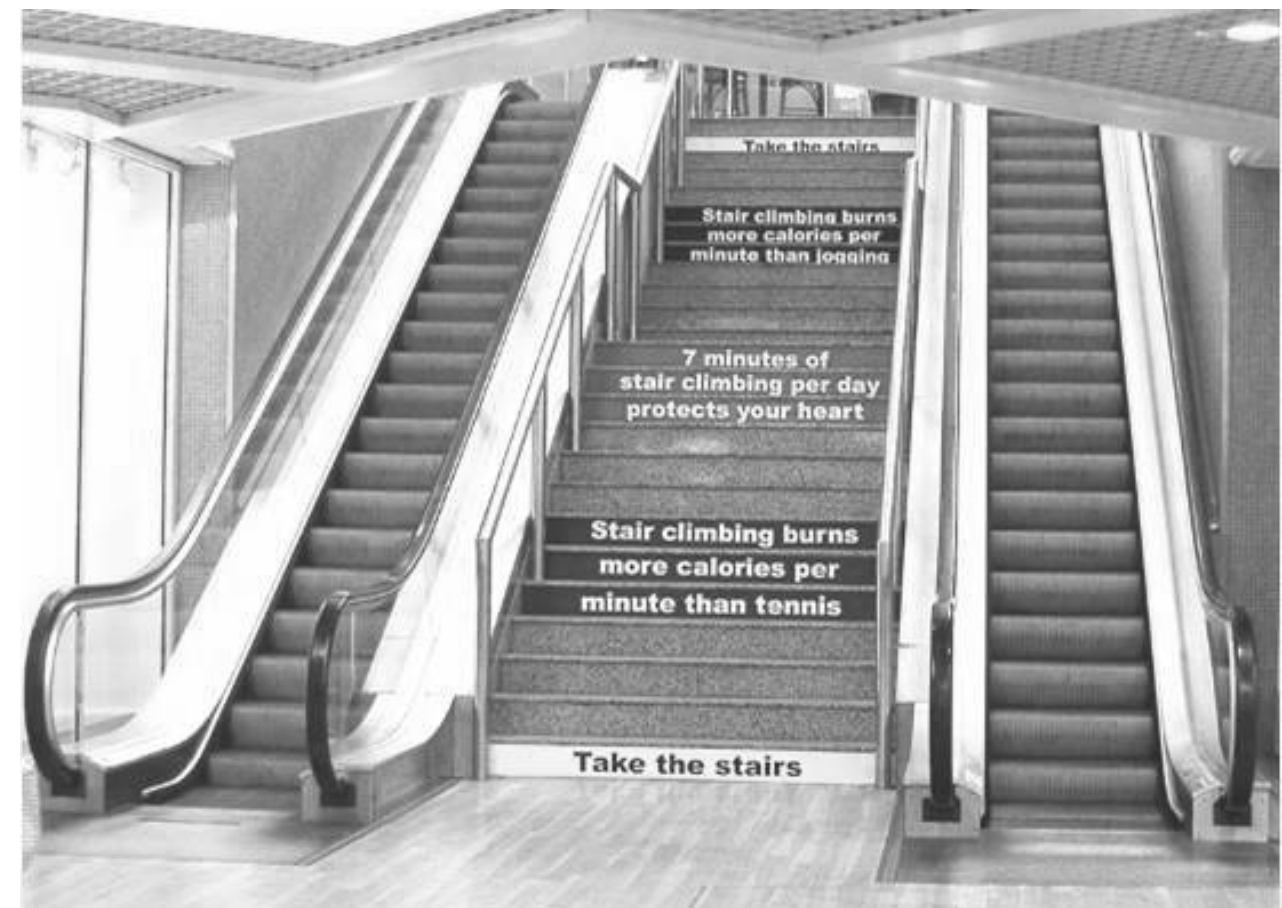

Figure 1. An archetypal public-access 'point-of-choice' (POC) from the study by Webb and Eves (2007). This is a 'true' POC, comprising immediately adjacent stairs and escalators. Banner prompts are attached to the stair risers. 
Public-access interventions are more frequently successful than work/community interventions (27/31 vs. 3/10) and produce larger absolute increases in rates of stair climbing (maximum $=10.6 \%$ vs. 9.2\%) [Nocon et al., 2010]. An explanation is that random waiting time associated with elevators dilutes potential effects, whereas escalators are continuously available (Eves et al., 2006). Given encouraging evidence for public-access interventions, health agencies have funded regional campaigns; the British Heart Foundation installed prompts throughout the London underground. It is now important to establish the feasibility of nationwide campaigns.

High footfall in public-access venues affords large audiences (Andersen et al., 1998). Importantly, intervention effects may also 'generalise'. Mall-goers who saw prompts at one POC chose stairs, unprompted, at a second ‘unadorned’ POC (Webb and Eves, 2007). Thus, prompts at one well-patronised public-access POC (e.g. a train terminus) could reach many pedestrians, with some maintaining stair choice in subsequent environments (e.g. home/workplace). Nonetheless, in the solitary paper showing generalisation for stair climbing, POCs were just $25 \mathrm{~m}$ apart (Webb and Eves, 2007). Without knowing how well generalisation effects endure after exposure, and if they operate between different types of venue, benefits are theoretical. Public-access interventions featuring follow-up phases also add little certainty regarding residual effects. Three months after prompts were removed Blamey et al. (1995) and Webb and Eves (2007) observed a decline in stair choice, whilst Brownell et al. (1980) observed complete reversion to baseline levels. These interventions may have been too short ( $\leq 13$ weeks) to establish lasting effects. Nevertheless, the evidencebased prediction must be that pedestrians can be persuaded to choose stairs when directly prompted. Nationwide campaigns would, therefore, require intervention at numerous POCs.

One might assume that public-access sites provide abundant POCs. Andersen et al. (1998) predicted that their successful intervention could be iterated in all 1,850 US malls, sparking 1.6 million extra daily ascents. A critical caveat must be added to these attractive forecasts. All public-access interventions featured 'true' POCs between immediately adjacent stairs/escalators, bar one, where stairs were still visible from the escalator in a 'quasi' POC (Lewis and Eves, 2011). Whilst public-access venues may be commonplace, it is unknown how many true/quasi POCs they harbour. Consequently, we audited POCs in public-access sites across England. 


\section{Method}

Urban areas $(\mathrm{N}=25)$ were selected, based on accessibility to investigators. Political boundaries for each were established from Ordinance Survey maps (http://www.ordancesurvey.co.uk/oswebsite/opendata/viewer/), which showed airports, tram stops and under/over-ground railway stations (tourist railways were excluded). Malls and bus stations were identified from commercial listings (The Retail Directory, 2010) and local authority websites, respectively. All public-access interventions to-date occurred in these venue types. Population statistics were derived from the 2001 Census, which used identical boundaries.

From September 2010-March 2011 two investigators visually inspected all venues, counting true POCs and quasi POCs (i.e. stairways visible in a $360^{\circ}$ plain on approach to the ascending escalator). Areas beyond airport security controls were not audited. At each POC, stairs were counted.

\section{Results}

The audited areas house 6,829,874 people, or 13.9\% of England's population. Table 1 stratifies results by venue type and area. Of 410 venues, 19 (5\%), had $\geq 1$ true POC and 12 (3\%) had $\geq 1$ quasi POC. Aggregating across areas/venues, there was a true POC and quasi POC for every 243,924 and 379,437 people, respectively.

There was regional variation; Doncaster provided five true/quasi POCs, whereas two similarly populated areas (Wakefield, Wirral) featured none. POCs were more common in airports (4/6 venues) than malls (11/85) and train stations (4/215). Median step count at POCs was 30 (range=15-41). Inter-observer agreement for POC classification was 100\%. 


\begin{tabular}{|c|c|c|c|c|c|c|c|c|c|c|c|c|c|c|c|c|c|c|c|c|c|c|c|}
\hline \multirow[b]{2}{*}{ Area } & \multirow[b]{2}{*}{ Тур } & \multirow[b]{2}{*}{ Pop. } & \multicolumn{3}{|c|}{ Airports } & \multicolumn{3}{|c|}{ Bus stations } & \multicolumn{3}{|c|}{$\begin{array}{l}\text { Over ground } \\
\text { rail stations }\end{array}$} & \multicolumn{3}{|c|}{$\begin{array}{l}\text { Underground } \\
\text { rail stations }\end{array}$} & \multicolumn{3}{|c|}{ Tram stops } & \multicolumn{3}{|c|}{$\begin{array}{c}\text { Shopping } \\
\text { malls }\end{array}$} & \multicolumn{3}{|c|}{$\begin{array}{l}\text { All venues } \\
\text { combined }\end{array}$} \\
\hline & & & $\mathbf{N}$ & $\stackrel{\varrho}{E}$ & $\begin{array}{l}\bar{y} \\
\overrightarrow{0} \\
\tilde{\sigma}\end{array}$ & $\mathbf{N}$ & $\stackrel{g}{E}$ & $\begin{array}{l}\cdot \bar{y} \\
\bar{\sigma}\end{array}$ & $\mathbf{N}$ & $\stackrel{\mathscr{E}}{E}$ & $\begin{array}{l}\cdot \vec{g} \\
\tilde{\sigma}\end{array}$ & $\mathbf{N}$ & $\stackrel{\mathscr{E}}{E}$ & 离 & $\mathbf{N}$ & $\stackrel{\underline{E}}{E}$ & $\begin{array}{l}\cdot \bar{y} \\
\tilde{z}\end{array}$ & $\mathbf{N}$ & $\stackrel{\mathscr{E}}{E}$ & $\stackrel{\cdot \vec{y}}{\tilde{\sigma}}$ & $\mathbf{N}$ & $\stackrel{\mathscr{E}}{E}$ & 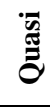 \\
\hline Bradford & a & 467665 & 0 & - & - & 1 & 0 & 0 & 18 & 2 & 0 & 0 & - & - & 0 & - & - & 2 & 0 & 2 & 21 & 2 & 2 \\
\hline Cambridge & $\mathrm{b}$ & 108863 & 0 & - & - & 1 & 0 & 0 & 1 & 0 & 0 & 0 & - & - & 0 & - & - & 3 & 0 & 0 & 5 & 0 & 0 \\
\hline Coventry & a & 300848 & 0 & - & - & 1 & 0 & 0 & 3 & 0 & 0 & 0 & - & - & 0 & - & - & 4 & 1 & 2 & 8 & 1 & 2 \\
\hline Derby & c & 221708 & 0 & - & - & 1 & 0 & 0 & 3 & 0 & 0 & 0 & - & - & 0 & - & - & 2 & 0 & 1 & 6 & 0 & 1 \\
\hline Doncaster & $\mathrm{a}$ & 286866 & 1 & 2 & 0 & 1 & 0 & 0 & 9 & 0 & 0 & 0 & - & - & 0 & - & - & 2 & 1 & 2 & 13 & 3 & 2 \\
\hline Exeter & $\mathrm{b}$ & 111076 & 0 & - & - & 1 & 0 & 0 & 7 & 0 & 0 & 0 & - & - & 0 & - & - & 2 & 0 & 0 & 10 & 0 & 0 \\
\hline Huddersfield & a & 388567 & 0 & - & - & 2 & 0 & 0 & 19 & 0 & 0 & 0 & - & - & 0 & - & - & 4 & 0 & 0 & 25 & 0 & 0 \\
\hline Leeds & a & 715402 & 1 & 1 & 0 & 1 & 0 & 0 & 16 & 3 & 1 & 0 & - & - & 0 & - & - & 14 & 4 & 1 & 32 & 8 & 2 \\
\hline Leicester & c & 279921 & 0 & - & - & 1 & 0 & 0 & 2 & 0 & 0 & 0 & - & - & 0 & - & - & 5 & 0 & 1 & 8 & 0 & 1 \\
\hline Liverpool & a & 439473 & 1 & 2 & 0 & 3 & 0 & 0 & 14 & 0 & 0 & 4 & 1 & 0 & 0 & - & - & 7 & 2 & 0 & 29 & 5 & 0 \\
\hline Milton Keynes & c & 207057 & 0 & - & - & 1 & 0 & 0 & 6 & 0 & 0 & 0 & - & - & 0 & - & - & 4 & 0 & 3 & 11 & 0 & 3 \\
\hline Norwich & $\mathrm{b}$ & 121550 & 1 & 0 & 0 & 1 & 0 & 0 & 1 & 0 & 0 & 0 & - & - & 0 & - & - & 2 & 0 & 1 & 5 & 0 & 1 \\
\hline Nottingham & c & 266988 & 0 & - & - & 1 & 0 & 0 & 2 & 0 & 0 & 0 & - & - & 21 & 0 & 0 & 5 & 1 & 0 & 29 & 1 & 0 \\
\hline Peterborough & c & 156061 & 0 & - & - & 1 & 0 & 0 & 4 & 0 & 0 & 0 & - & - & 0 & - & - & 5 & 0 & 0 & 10 & 0 & 0 \\
\hline Scunthorpe & c & 152849 & 1 & 0 & 0 & 1 & 0 & 0 & 12 & 0 & 0 & 0 & - & - & 0 & - & - & 1 & 0 & 0 & 15 & 0 & 0 \\
\hline Sheffield & a & 513234 & 0 & - & - & 1 & 0 & 0 & 6 & 0 & 0 & 0 & - & - & 48 & 0 & 0 & 5 & 0 & 1 & 60 & 0 & 1 \\
\hline Solihull & a & 199517 & 1 & 1 & 0 & 0 & - & - & 11 & 3 & 0 & 0 & - & - & 0 & - & - & 3 & 0 & 1 & 15 & 4 & 1 \\
\hline St. Helens & a & 176843 & 0 & - & - & 1 & 0 & 0 & 10 & 0 & 0 & 0 & - & - & 0 & - & - & 2 & 0 & 0 & 13 & 0 & 0 \\
\hline Stoke-on-Trent & c & 240636 & 0 & - & - & 1 & 0 & 0 & 3 & 0 & 0 & 0 & - & - & 0 & - & - & 1 & 2 & 1 & 5 & 2 & 1 \\
\hline Wakefield & $\mathrm{a}$ & 315172 & 0 & - & - & 3 & 0 & 0 & 16 & 0 & 0 & 0 & - & - & 0 & - & - & 1 & 0 & 0 & 20 & 0 & 0 \\
\hline Warrington & c & 191080 & 0 & - & - & 1 & 0 & 0 & 6 & 0 & 0 & 0 & - & - & 0 & - & - & 2 & 0 & 0 & 9 & 0 & 0 \\
\hline Widnes \& Runcorn & c & 118208 & 0 & - & - & 1 & 0 & 0 & 4 & 0 & 0 & 0 & - & - & 0 & - & - & 1 & 0 & 0 & 6 & 0 & 0 \\
\hline Wigan & a & 301415 & 0 & - & - & 1 & 0 & 0 & 10 & 0 & 0 & 0 & - & - & 0 & - & - & 3 & 0 & 0 & 14 & 0 & 0 \\
\hline Wirral & a & 312293 & 0 & - & - & 0 & - & - & 25 & 0 & 0 & 2 & 0 & 0 & 0 & - & - & 3 & 1 & 0 & 30 & 1 & 0 \\
\hline Wolverhampton & $\mathrm{a}$ & 236582 & 0 & - & - & 1 & 0 & 0 & 1 & 0 & 0 & 0 & - & - & 7 & 0 & 0 & 2 & 1 & 1 & 11 & 1 & 1 \\
\hline TOTAL & & 6829874 & 6 & 6 & 0 & 28 & 0 & 0 & 209 & 8 & 1 & 6 & 1 & 0 & 76 & 0 & 0 & 85 & 13 & 17 & 410 & 28 & 18 \\
\hline
\end{tabular}

Table 1. Number of 'true' and 'quasi' point-of-choice (POCs) found in 25 urban areas in England (September 2010 - March 2011), stratified by venue type 


\section{Discussion}

In England the availability of public-access POCs is sporadic, even when little-tested quasi POCs are included. This precludes nationwide campaigns. Instead, interventions should be considered locally, based on available sites. Distribution of POCs across venue types is also noteworthy. Proportionately, POCs are most common in airports, where many individuals are occasional visitors, and least common in bus/train/tram stations, where reexposure may occur.

Whilst public-access settings offer few POCs, there are probably stairs somewhere on the premises, to which pedestrians could be directed. According to work/community studies, however, interventions are less effective where stairs are remotely located. One study introduced prompts in five settings (Bungum et al., 2007). The magnitude of effects depended on visibility of the stairs from the elevator. Elsewhere, identical prompts were introduced at four university buildings; two where stairs were visible from the elevator and two where stairs were hidden (Grimstvedt et al., 2010). Proportionate to baseline stair use, analyses indicated consistent effects across sites. Uneven baseline levels were, however, a complicating factor. Raw figures show appreciably greater effects at the 'visible' $(43.6 \% \rightarrow 59.4 \%)$ versus 'hidden' stairwells $(13.2 \% \rightarrow 22.9 \%)$. Unlike mass media campaigns, stair climbing interventions offer immediate opportunities to convert healthy intentions into action. These are compromised if individuals must seek out concealed staircases.

Nationwide campaigns may be better channelled through more abundant work/community settings. Many pedestrians would be repeat visitors (e.g. employees/students), such that effects might become re-enforced. Moreover, investigators have improved upon lacklustre effects of early work/community interventions, by refining message content (Eves et al., 2006). Encouragingly, heightened effects on stair climbing have been achieved in overweight (+5.4\%) vs. normal-weight (+2.5\%) office workers (Eves et al., 2006). Despite reasons for optimism, evidence is limited regarding longevity of effects, relative efficacy in sub-groups (e.g. men vs. women [Coleman and Gonzalez, 2001; Eves et al., 2006]), optimal message content, value of culturally-tailored prompts and benefits of additional initiatives (e.g. stairwell renovation). Thus, a definitive protocol for work/community interventions is presently unavailable. 
Our findings have methodological implications. All stair climbing interventions used interrupted time-series designs, whereby behaviour at one site was monitored during baseline and subsequent intervention phases. Whilst investigators controlled for common confounders (e.g. demographics), critics contend that without control/comparison conditions, findings are unconvincing. They recommend quasi-randomized designs, whereby paired intervention/control sites are matched across all additional moderators of stair choice. In practice, moderators are numerous and show considerable site-to-site variation. They include demographics (Andersen et al., 1999; Blamey et al., 1995; Brownell et al., 1980; Bungum et al., 2007; Eves et al., 2006; Grimstvedt et al., 2010; Lewis and Eves, 2011; Webb and Eves, 2007); spatial characteristics (e.g. stairwell height [Webb and Eves, 2007]); 'pedestrian volume’ (Lewis and Eves, 2011; Webb and Eves, 2007); 'behavioural’ context (e.g. leisure/commuter) and presence of stair climbing 'models' (Adams et al., 2006). The limited pool of public-access POCs suggests that in England, at least, quasi-randomization may be unattainable.

This audit was not exhaustive. Stair/escalator pairings may be found elsewhere (e.g. inside shops/banks [Coleman and Gonzalez, 2001]). It is unclear, however, if such establishments would host large-scale campaigns. Areas were also chosen at convenience. They are, however, distributed across England, with little reason to question their representativeness. Finally, results only apply to England. Local auditing appears essential to obtain country-specific information on availability of public-access POCs.

\section{Conclusion}

The limited number of POCs in English public-access settings suggests that national stair climbing campaigns may be better channelled through work/community venues.

\section{Conflict of Interests Statement:}

The authors declare that there are no conflicts of interest.

\section{Acknowledgements:}




\section{References}

Adams, M., Hovell, M., Irvin, V., Sallis, J., Coleman, K., Liles, S., 2006. Promoting stair use by modeling: an experimental application of the behavioral ecological approach. Am. J. Health Promot. 21, 101-109.

Andersen, R.E., Franckowiak, S.C., Snyder, J., Bartlett, S.J., Fontaine, K.R., 1998. Can inexpensive signs encourage the use of stairs? Results of a community intervention. Ann. Intern. Med. 129, 363-369.

Blamey, A., Mutrie, M., Aitchison, T., 1995. Health promotion by encouraged use of stairs. Br. Med. J. 311, 289-290.

Brownell, K.D., Stunkard, A.J., Albaum, J.M., 1980. Evaluation and modification of exercise patterns in the natural environment. Am. J. Psychiatry. 137, 1540-1545.

Bungum, T., Meacham, M., Truax, N., 2007. The effects of signage and the physical environment on stair usage. J. Phys. Act. Health. 4, 237-244.

Coleman, K.J., Gonzalez, E.C., 2001. Promoting stair use in a US-Mexico border community. Am. J. Public Health. 91, 2007-2009.

Eves, F.F., Webb, O.J., 2006. Worksite interventions to promote stair climbing; reasons for caution. Prev. Med. 43, 4-7.

Grimstvedt, M.E., Kerr, J., Oswalt, S.B., Fogt, D.L., Vargas-Tonsing, T.M., Yin, Z., 2010. Using signage to promote stair use on a university campus in hidden and visible stairwells. J. Phys. Act. Health. 7, 232-238. 
Lewis, A.L., Eves, F.F., 2011. Specific effects of a calorie-based intervention on stair climbing in overweight commuters. Ann Behav Med. DOI: 10.1007/s12160-011-9283-z

Meyer, P., Kayser, B., Kossovsky, M.P. et al., 2010. Stairs instead of escalators at workplace: cardioprotective effects of a pragmatic intervention. Eur. J. Cardiovasc. Prev. Rehabil. 17, 569-575.

Nocon, M., Müller-Riemenschneider, F., Nitzschke, K., Willich, S.N., 2010. Increasing physical activity with point-of-choice prompts - a systematic review. Scand. J. Public Health. 38, 633-638.

Webb, O.J., Eves, F.F., 2007. Promoting stair climbing: intervention effects generalize to a subsequent stair ascent. Am. J. Health Promot. 22, 114-119. 